\title{
Dynamic analysis of an orthotropic viscoelastic cylindrical panel of variable thickness
}

\author{
Mirziyod Mirsaidov ${ }^{1}$, Rustamkhan Abdikarimov ${ }^{2}$, Bakhodir Normuminov ${ }^{1}$ and Dadakhan \\ Khodzhaev $^{1 *}$ \\ ${ }^{1}$ Tashkent Institute of Irrigation and Agricultural Mechanization Engineers, Tashkent, Uzbekistan \\ ${ }^{2}$ Tashkent Institute of Finance, Tashkent, Uzbekistan
}

\begin{abstract}
The intensive development of the modern industry is associated with the emergence of a variety of new composite materials. Plates, panels, and shells of variable thickness made of such materials are widely used in engineering and machine building. Modern technology for the manufacture of thin-walled structures of any configuration makes it possible to obtain structures with a given thickness variation law. Such thin-walled structures are subjected to various loads, including periodic ones. Nonlinear parametric vibrations of an orthotropic viscoelastic cylindrical panel of variable thickness are investigated without considering the elastic wave propagation. To derive a mathematical model of the problem, the Kirchhoff-Love theory is used in a geometrically nonlinear setting. The viscoelastic properties of a cylindrical panel are described by the hereditary Boltzmann-Volterra theory with a three-parameter Koltunov-Rzhanitsyn relaxation kernel. The problem is solved by the Bubnov-Galerkin method in combination with the numerical method. For the numerical implementation of the problem, an algorithm and a computer program were developed in the Delphi algorithmic language. Nonlinear parametric vibrations of orthotropic viscoelastic cylindrical panels under external periodic load were investigated. The influence of various physical, mechanical, and geometric parameters on the panel behavior, such as the thickness, viscoelastic and inhomogeneous properties of the material, external periodic load, were studied.
\end{abstract}

\section{Introduction}

In modern technology and construction, structures such as plates, panels, and shells made of composite materials are increasingly used. Modern methods and technologies for their manufacture make it possible to obtain designs of any known predetermined shapes and sizes. Often, such structures are subjected during operation to various static and dynamic loads. Among such external loads acting on structures, periodic loads are of particular interest. In the classical works of V. Bolotin [1] and A.Volmir [2], various dynamic problems of elastic systems are considered. Special attention is paid to the vibrations of the system under periodic loads.

*Corresponding author: dhodjaev@mail.ru 
The theory of vibrations and dynamic stability of systems was developed in the studies of M.Amabili [3].

In the literature, there are several publications devoted to the study of nonlinear vibrations and dynamic stability of plates, panels, and shells of constant thickness. An overview of the studies conducted in 2003-2013 is given in [4]. It presents the results of theoretical and experimental studies devoted to specific dynamic problems.

In [5], based on Donnell's theory, the results of studying the influence of initial irregularities on parametric vibrations of geometrically nonlinear cylindrical shells are presented.

Studying the dynamic stability of composite plates under periodic loads is given in [6]. The Bolotin method is used to construct the regions of dynamic instability.

The study of composite plates subjected to periodic compressive loads is considered in [7]. Based on the finite element method, the Mathieu-Hill equation is solved. The regions of dynamic instability of the plates are constructed.

The study results of nonlinear vibrations of composite plates under periodic loads are given in $[8,9]$. Based on the Galerkin method, equations of motion of the Karman type are solved.

In [10], the parametric instability of composite plates is investigated. Based on the Bolotin method, the influence of the plate geometry, boundary conditions, and load intensity on the plate behavior is shown.

Parametric vibrations of layered plates are investigated in [11] based on the KirchhoffLove theory.

The study in [12] is devoted to the parametric vibrations of viscoelastic plates based on the generalized Hamiltonian theory. The influence of the viscoelastic properties of the material on the dynamic instability region is shown.

There are many publications devoted to studying the stability of plates and shells of variable thickness under various compressive loads.

Vibrations of various types of composite plates and shells of variable thickness are investigated in [13]. The results obtained based on the proposed method are compared with the results of other researchers, obtained analytically and numerically.

In $[14,15]$, based on the Bolotin method, the problem of dynamic instability of composite plates of variable thickness is solved. The influence of the geometrical and physical-mechanical parameters of the plate on the dynamic instability region is shown.

In [16] the dynamic stability of composite rectangular panels under various compressive loads is investigated based on the finite element method. The problem is reduced to solving the Mathieu-Hill equation.

An analysis of the literature sources shows that little attention is paid to the study of parametric vibrations of orthotropic viscoelastic plates, panels, and shells of variable thickness [17-19].

In the current article, the authors investigate parametric vibrations of an orthotropic viscoelastic cylindrical panel of variable thickness without considering elastic wave propagation in a geometrically nonlinear formulation.

\section{Material and Methods}

Consider an orthotropic viscoelastic cylindrical panel with sides $a$ and $b$, variable thickness $h=h(x)$, and radius of curvature $R$ under the action of an external periodic load $P(t)=P_{0}+P_{1} \cos \Theta t$.

Similar to [2], introducing the stress function $\Phi$ into equation [20], we obtain the equation of motion of an orthotropic viscoelastic cylindrical panel relative to the deflection $w$ and the stress function $\Phi$. 
The solution to the resulting system is sought in the following form,

$$
w(x, y, t)=\sum_{n=1}^{N} \sum_{m=1}^{M} w_{n m}(t) \psi_{n m}(x, y), \Phi(x, y, t)=\sum_{n=1}^{N} \sum_{m=1}^{M} \Phi_{n m}(t) \chi_{n m}(x, y)
$$

where: $w_{n m}=w_{n m}(t)$ and $\Phi_{n m}=\Phi_{n m}(t)$ are the required functions of time; $\psi_{n m}(x, y)$, $\chi_{n m}(x, y), \quad n=1,2, \ldots, N ; m=1,2, \ldots, M$ are the coordinate functions that satisfy the given boundary conditions of the problem.

Substituting (1) into the original equation, performing the Bubnov-Galerkin procedure, introducing dimensionless quantities, and keeping the previous notation with respect to unknowns $w_{n m}=w_{n m}(t), \Phi_{n m}=\Phi_{n m}(t)$, we obtain a system of nonlinear integrodifferential equations with corresponding initial and boundary conditions.

Integrating the system of resolving equations of an orthotropic viscoelastic cylindrical panel of variable thickness twice in $t$, it can be written in integral form. Then, according to the numerical method proposed in [21], assuming that $t=t_{i}, t_{i}=i \Delta t, i=1,2, \ldots$ ( $\Delta t$ is the integration step) and replacing the integrals with quadrature trapezoidal formulas for calculating unknowns $w_{\text {inm }}=w_{\text {inm }}\left(t_{i}\right), \Phi_{\text {inm }}=\Phi_{\text {inm }}\left(t_{i}\right)$, we obtain a recurrent formula.

Here, a weakly singular Koltunov-Rzhanitsyn kernel of the following form is used as a relaxation kernel.

$$
\Gamma(t)=A e^{-\beta t} \cdot t^{\alpha-1}, \quad A>0, \quad \beta>0, \quad 0<\alpha<1
$$

\section{Results and Discussion}

To implement the solution algorithm, a program was developed in the Delphi algorithmic language. The results of calculations, with various physical and geometrical parameters of an orthotropic viscoelastic cylindrical panel of variable thickness, are shown in a graphic form. As initial data, the following values were taken in calculations: $A, A_{i j}=0.05 ; \delta=25$; $q=0 ; \lambda=1 ; k_{y}=20 ; r=0 ; \delta_{0}=0.3 ; \delta_{1}=0.5 ; \Theta=1.1$.

The thickness variation law is set analytically and can be of any form. Let the panel thickness change according to the following law $h(x)=\frac{1}{2} h_{0}[1+\varepsilon \sin (2 r-1) \pi x]$, where $r$ is the parameter characterizing the thickness variability; $h_{0}$ is the panel thickness corresponding to $r=0.5$.

Figure 1 shows the profiles of the thickness change for various values of parameter $r$. 

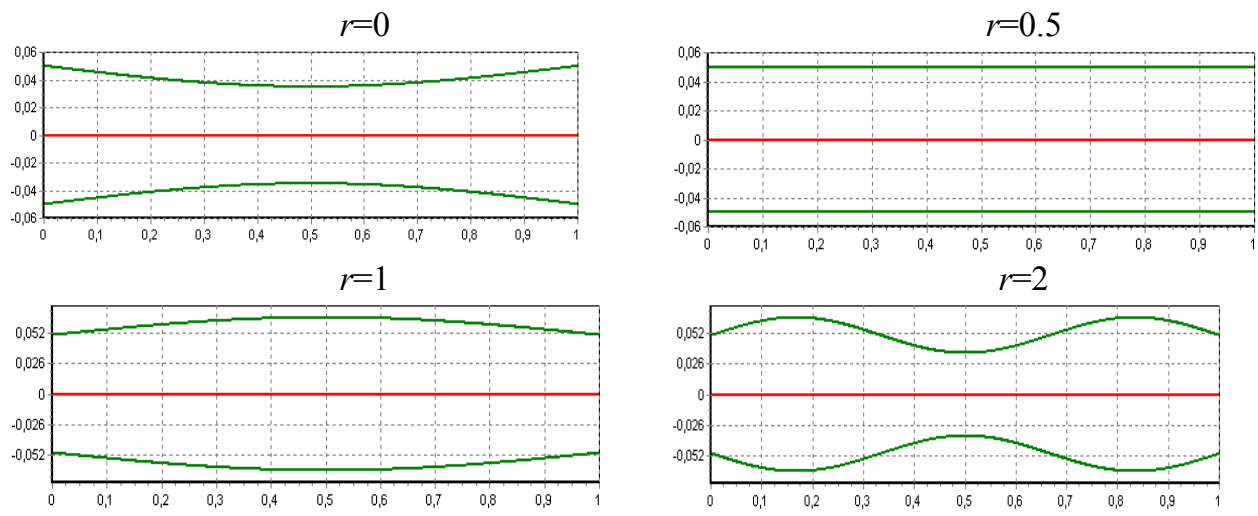

Fig. 1. Profiles of the thickness change

The influence of the viscoelastic properties of the panel material on its behavior was studied (Figure 2). The results obtained show that viscoelastic properties lead to a decrease in the vibration amplitude.

The vibration modes of an orthotropic elastic and viscoelastic cylindrical panel corresponding to this case are shown in Figure 3.

Figure 4 shows the change in the deflection of a cylindrical panel as a function of time for different values of the thickness variability parameter $r$. It can be seen that an increase in panel stiffness leads to a phase shift of vibrations.

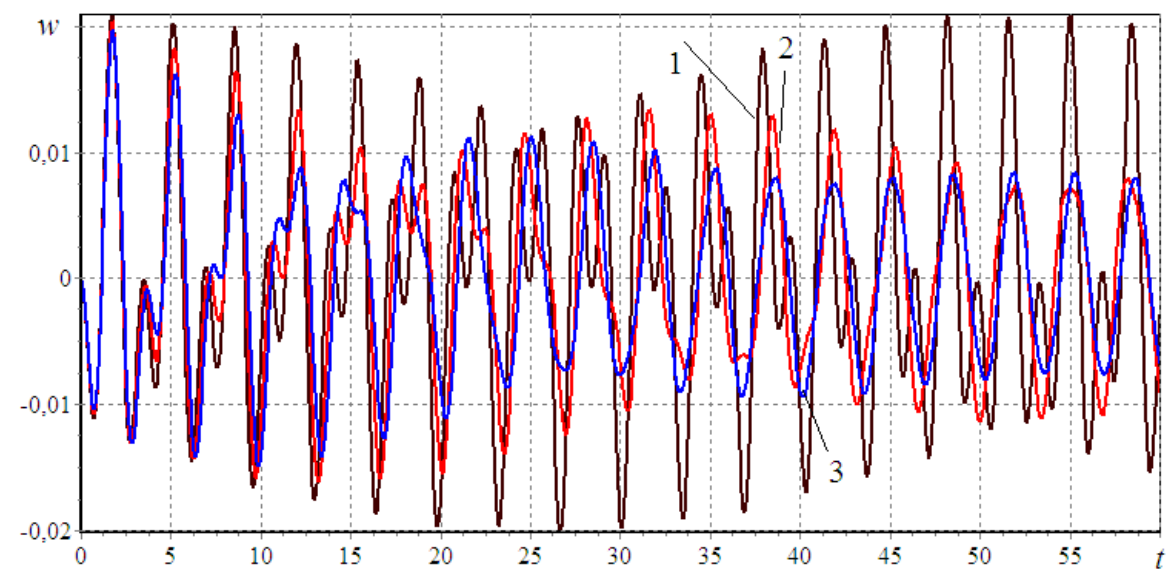

Fig. 2. Deflection versus time at: 1) $A, A_{i j}=0$; 2) $A, A_{i j}=0.05$; 3) $A, A_{i j}=0.1$ 


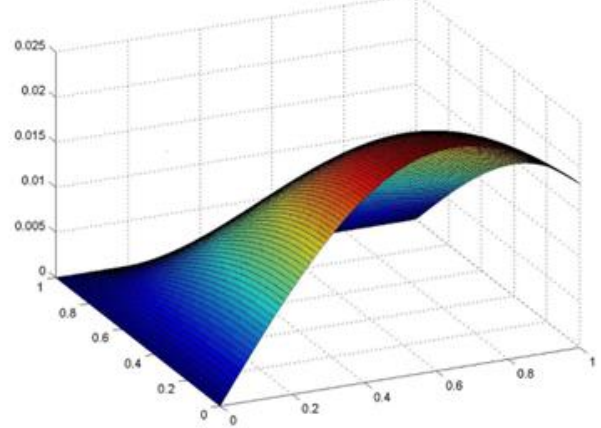

a)

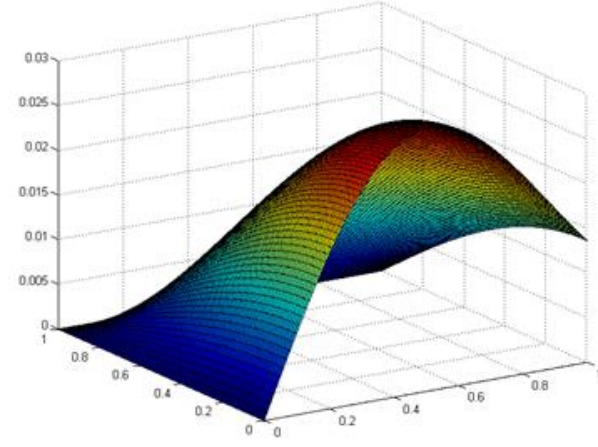

b)

Fig.3. Vibration mode of a cylindrical panel of variable thickness at $t=4$ : a) elastic; b) viscoelastic

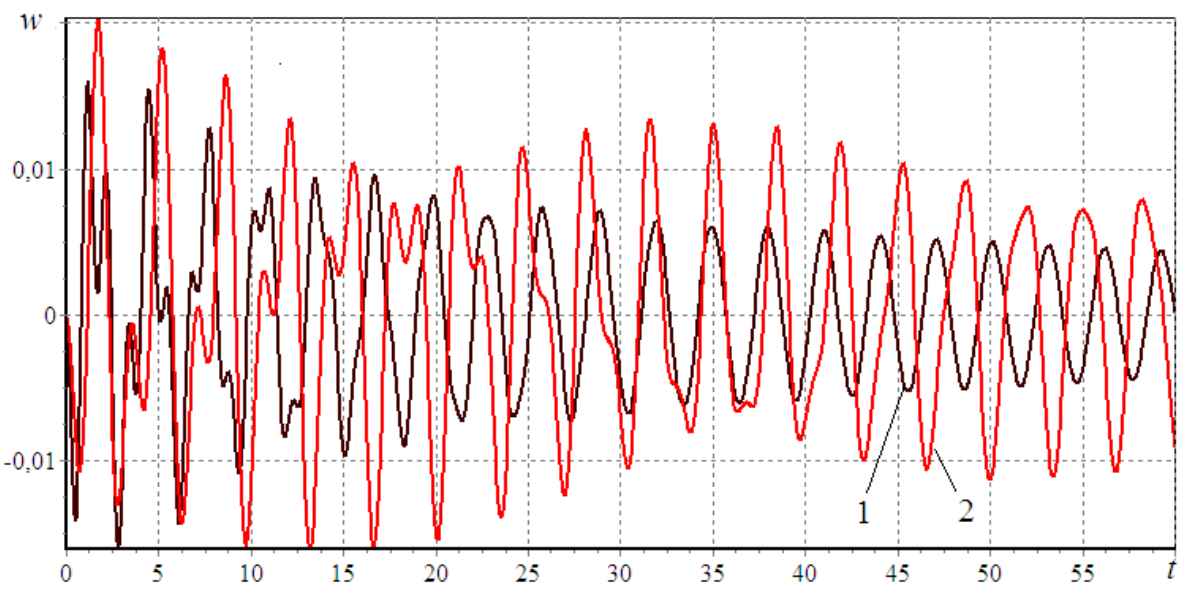

Fig. 4. Deflection versus time: 1$)$ constant thickness $(r=0.5) ; 2)$ variable thickness $(r=0)$

The vibration modes of the panel corresponding to this case are shown in Fig. 5.

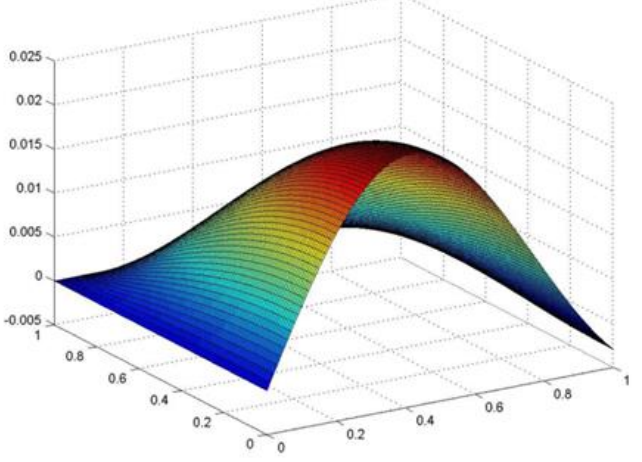

a)

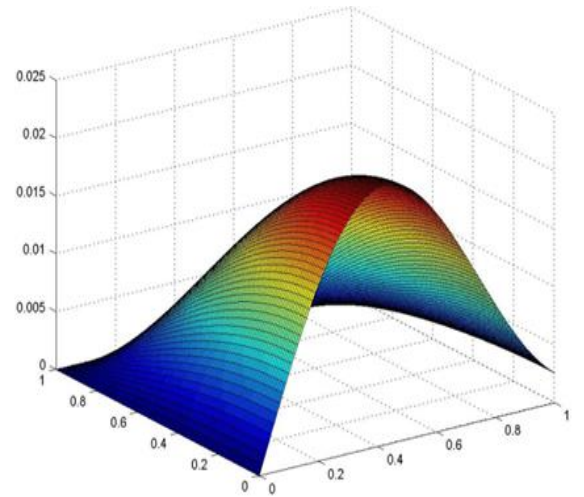

b)

Fig. 5. Vibration mode of a cylindrical panel at $t=20$ : 1) constant thickness $(r=0.5)$; 2$)$ variable thickness $(r=0)$

Figure 6 shows the results of studying the panel behaviour at various values of the curvature $k y$. An analysis of the results shows that an increase in this parameter leads to an 
increase in the amplitude of oscillations and a phase shift.

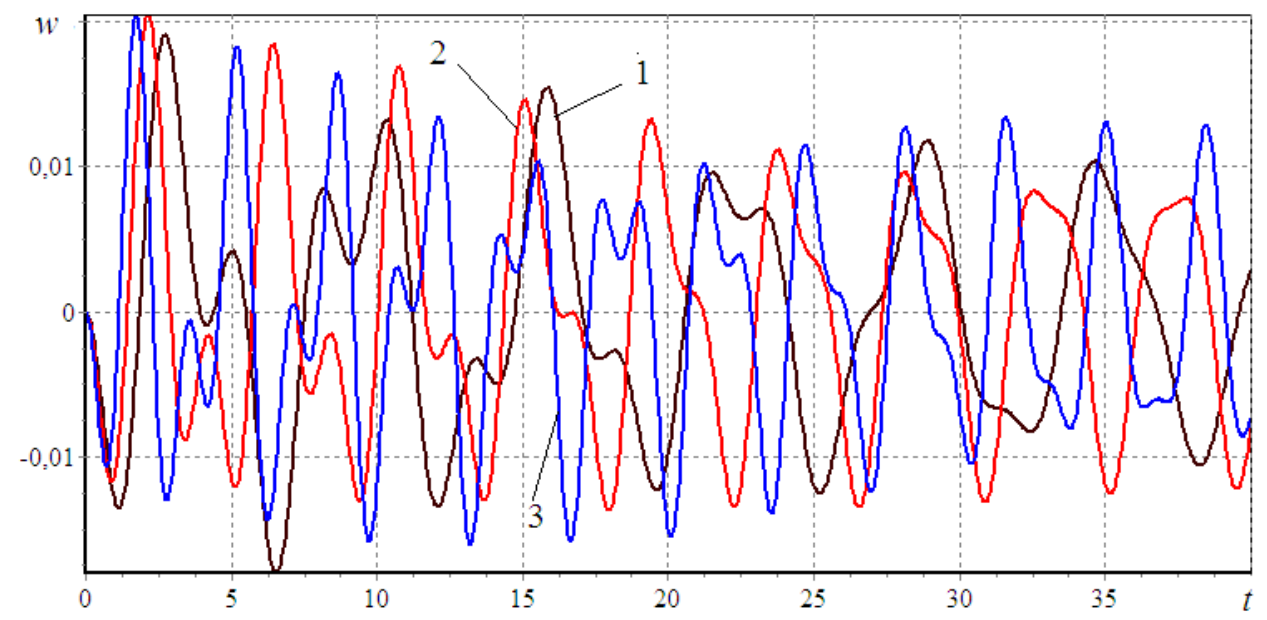

Fig. 6. Deflection versus time at: $k y=10(1) ; 15(2) ; 20$ (3)

\section{Conclusions}

Parametric vibrations of an orthotropic viscoelastic cylindrical panel of variable thickness are investigated in a geometrically nonlinear formulation without considering the elastic wave propagation.

A mathematical model, method, and computer program for the study of parametric vibrations of viscoelastic orthotropic cylindrical panels of variable thickness without considering the elastic wave propagation were developed.

The effect on the amplitude-time characteristics of changes in the physical-mechanical and geometrical parameters of the panel material is estimated.

The method proposed in the article can be used for various thin-walled structures, such as plates, panels, and shells of variable thickness.

\section{References}

1. V. V. Bolotin, The Dynamic Stability of Elastic Systems (Holden-Day, San Francisco, 1964)

2. A. S. Volmir, Stability of Deformable Systems (NASA AD, 1965)

3. M. Amabili, Nonlinear Vibrations and Stability of Shells and Plates (Cambridge University Press, 2008)

4. F. Alijani and M. Amabili, Int. J. Non. Linear. Mech. 58, 233 (2014)

5. R. Kochurov and K. V. Avramov, Int. J. Solids Struct. 49, 537 (2012)

6. W.-R. Chen, C.-S. Chen, and J.-H. Shyu, Appl. Math. Comput. 223, 127 (2013)

7. Z. Yusof and Z. A. Rasid, AIP Conf. Proc. 1750, 30044 (2016)

8. M. Darabi and R. Ganesan, Compos. Struct. 176, 82 (2017)

9. M. Darabi and R. Ganesan, Nonlinear Dyn. 91, 187 (2018)

10. H. Wu, J. Yang, and S. Kitipornchai, Int. J. Mech. Sci. 135, 431 (2018)

11. V. Stojanović, M. D. Petković, and J. Deng, Compos. Part B Eng. 167, 631 (2019)

12. D.-B. Zhang, Y.-Q. Tang, and L.-Q. Chen, Eur. J. Mech. - A/Solids 75, 142 (2019)

13. M. Bacciocchi, M. Eisenberger, N. Fantuzzi, F. Tornabene, and E. Viola, Compos. Struct. 156, 218 (2016) 
14. M. A. R. Loja, J. I. Barbosa, and C. M. Mota Soares, Compos. Struct. 182, 402 (2017)

15. M. A. R. Loja and J. I. Barbosa, Compos. Struct. 237, 111905 (2020)

16. M. Rasool and M. K. Singha, Compos. Struct. 225, (2019)

17. M. M. Mirsaidov, R. A. Abdikarimov, N. I. Vatin, V. M. Zhgutov, D. A. Khodzhaev, and B. A. Normuminov, Mag. Civ. Eng. 82, 112 (2018)

18. D. Khodzhaev, B. Normuminov, Y. Mustapakulov, and A. Mottaeva, E3S Web Conf. 110, (2019)

19. B. Normuminov, R. Abdikarimov, D. Khodzhaev, and Z. Khafizova, IOP Conf. Ser. Mater. Sci. Eng. 896, (2020)

20. B. Normuminov, R. Abdikarimov, M. Mirsaidov, D. Khodzhaev, and B. Mirzaev, \{IOP $\}$ Conf. Ser. Mater. Sci. Eng. 869, 52034 (2020)

21. R. A. Abdikarimov and D. A. Khodzhaev, Mag. Civ. Eng. 49, 83 (2014) 